\title{
Independent Domination Number in Triangular \& Quadrilateral Snake graph
}

\author{
N.Senthurpriya , S.Meenakshi
}

\begin{abstract}
Let $V$ be the vertex set and $E$ be the edge set of a graph $G$, the vertex set $V$ has a subset $S$ such that $S$ contains vertices which is adjacent to atleast one vertex in $V$ which is not in $S$, then $S$ is said to be dominating set of $G$. If the vertex in $S$ is not adjacent to each other, then $S$ is said to be independent dominating set of $G$ and so $i(G)$ denotes the independent domination number, the minimum cardinality of an independent dominating set in $G$. In this paper, we obtain independent domination number for a triangular snake, alternate triangular snake, double triangular snake, alternate double triangular snake, quadrilateral snake, alternate quadrilateral snake, double quadrilateral snake and alternate double quadrilateral snake graphs.
\end{abstract}

Keywords: Alternate double triangular snake, alternate double quadrilateral snake, alternate quadrilateral snake, alternate triangular snake, double triangular snake, double quadrilateral snake, , quadrilateral snake, triangular snake.

\section{INTRODUCTION}

The dominating sets were initially started with the game of chess in India over 400 years ago, in which we study sets of chess pieces which cover different squares of the chessboard. Later the eight Queens and Five Queens problems again gave the interest in dominating concepts example, In the books of Ahrens in 1901 [16]. Finally, Berge in 1958 [2] and ore in 1962 [9] published the books related to the topic domination which gave proper mathematical definition but by 1972 Cockayne and Hedetniemi [3,4] again gone through domination and began to study it, ultimately in 1975 a survey of the results were published and the independent domination number were introduced and it is denoted in the form $i(G)$. This rekindled the researchers to work on it. Goddard et al. [15], Kostichka [1] and Lam et al. [10] researched much about the independent dominating number in regular graph and cubic graph. Favaron [8] studied the sharp upper bounds of $\boldsymbol{i}(\boldsymbol{G})$ for general graphs and the work was extended by Haviland [6]. Cockayne et al. [5] found the product of the $i(G)$ of a graph and its complement with its upper bound, while Shiu et al. [14] found the $i(G)$ of triangle-free graphs and characterizing the extremal graphs with its upper bounds.

\section{DEFINITIONS}

Revised Manuscript Received on December 5, 2019.

Correspondence Author

N.Senthurpriya, Department of mathematics, Vels institute of science technology and advanced studies, Pallavaram, Chennai, India. Email: pspriyasaha@gmail.com

S.Meenakshi, Department of mathematics, Vels institute of science technology and advanced studies, Pallavaram, Chennai, India. Email:meenakshikarthikeyan@yahoo.co.in
Definition 1. [11] For $v \in V(G)$, the open neighbourhood of $v$, denoted as $N_{G}(v)$, is the vertices of the set adjacent with a vertex $v$; and the closed neighbourhood of a vertex $v$, denoted by $N_{G}[v]$, is given by $N_{G}(v) \bigcup\{v\}$. For a set $S \subseteq V(G)$, the open neighbourhood of a set $\mathrm{S}$ is defined by $N_{G}(S)=\bigcup_{v \in S} N_{G}(v)$ and the closed neighbourhood of $\mathrm{S}$ is defined as $N_{G}[S]=N_{G}(S) \cup S$. For brevity, we denote $N_{G}(S)$ by $N(S)$ and $N_{G}[S]$ by $N[S]$.

Definition 2. [11] Let $\mathrm{G}$ be a graph with Vertex set $\mathrm{V}$ and edge set $\mathrm{E}$, let $\mathrm{S}$ be the subset of $\mathrm{V}$ such that every vertex in $\mathrm{V}$ which is not in $\mathrm{S}$ must contain atleast one neighbour in $\mathrm{S}$. The domination number of $\mathrm{G}$ is denoted by $\gamma(G)$, the minimum cardinality of dominating set of G.

Definition 3. [13] If $S$ is both an independent and dominating set of a graph $G$ then $S$ is said to be an independent dominating set of graph G. The independent domination number is denoted by $i(G)$, the minimum cardinality of an independent dominating set in G.

Definition 4. [12] Triangular snake:

The triangular snake $T_{n}$ is obtained from the path $P_{n}$ by replacing each edge of the path by a triangle $C_{7}$.

Definition 5. [12] Alternate triangular snake:

An alternate triangular snake $A\left(T_{n}\right)$ is obtained from a path $a_{1}, a_{2}, \ldots a_{n}$ by joining $a_{i}$ and $a_{i+1}$ (alternately) to a new vertex $v_{i}$.

Definition 6. [12] Double Triangular snake:

A double triangular snake $D\left(T_{n}\right)$ consists of two triangular snakes that have a common path.

Definition 7. [12] Double Alternate triangular snake:

A double alternate triangular snake $A\left(D\left(T_{n}\right)\right)$ consists of two alternate triangular snakes that have a common path.

Definition 8. [7] Quadrilateral snake:

A quadrilateral snake $Q_{n}$ is obtained from a path $a_{1}, a_{2}, \ldots a_{n}$ by joining $a_{i}$ and $a_{i+1}$ to new vertices $b_{i}$ and $c_{i}$ respectively and joining the vertices $b_{i}$ and $c_{i}$ for $\mathrm{i}=1,2, \ldots \mathrm{n}-1$. That is every edge of a path is replaced by a cycle $C_{4}$.

Definition 9. [7] Alternate quadrilateral snake:

An alternate quadrilateral snake $A\left(Q_{n}\right)$ is obtained from a path $a_{1}, a_{2}, \ldots a_{n}$ by joining $a_{i}$ and $a_{i+1}$ to new vertices $b_{i}$ and $c_{i}$ respectively and joining the vertices $b_{i}$ and $c_{i}$ for $\mathrm{i} \equiv 1(\bmod 2)$ and $\mathrm{I} \leq \mathrm{n}-1$ and then joining $b_{\mathrm{i}}$ and $c_{\mathrm{i}}$. That is every alternate edge of a path is replaced by a cycle $C_{4}$.

Definition 10. [7] Double Quadrilateral snake:

A double quadrilateral snake $D\left(Q_{n}\right)$ is obtained from two quadrilateral snakes that have a common path.

Definition 11. [7] Double Alternate quadrilateral snake: An alternate double quadrilateral snake $A\left(D\left(Q_{n}\right)\right)$ is obtained from two alternative quadrilateral snakes that have a common path. 


\section{Theorem 1:}

Let $T_{n}$ be the triangular snake obtained from the path $P_{n}$ by replacing each edge of the path by a triangle $C_{3}$ then $\mathrm{i}\left(T_{\mathrm{n}}\right)=\left\{\begin{array}{l}{\left[\frac{\mathrm{n}+1}{2}\right] \text { when } n \text { is odd }} \\ {\left[\frac{\mathrm{n}}{2}\right] \quad \text { when } n \text { is even }}\end{array}\right.$

Proof:

Let $a_{1}, a_{2}, \ldots a_{n}$ be the vertices of $T_{n}$ and $b_{i}$ be the new vertex joining $a_{i}$ and $a_{i+1}$ of a path by a triangle $C_{3}$.

Now we select the vertices for the set $S$ (independent domination set) from a graph $T_{n}$, in such a way that each vertex in $\mathrm{S}$ is adjacent to atleast one vertex in $\mathrm{V}-\mathrm{S}$ and that each vertex in $\mathrm{S}$ is independent.

Choosing such a way we get the independent domination number for $T_{\mathrm{n}}$ as (i.e.,)

$$
\mathrm{i}\left(T_{n}\right)=\left\{\begin{array}{l}
{\left[\frac{n+1}{2}\right\rceil \text { when } n \text { is odd }} \\
{\left[\frac{n}{2}\right] \quad \text { when } n \text { is even }}
\end{array}\right.
$$

for example, for $\mathrm{n}$ is odd

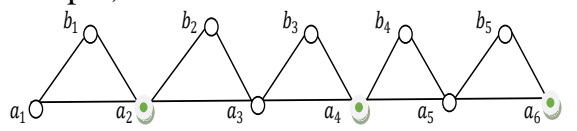

For $\mathrm{n}$ is even

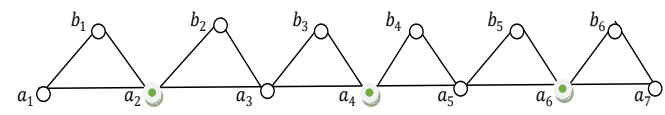

The highlighted vertices represent the independent domination set.

\section{Theorem 2:}

Let $A\left(T_{n}\right)$ be the alternate triangular snake obtained from the path $P_{\mathrm{n}}$ by replacing each alternate edge of the path by a triangle $C_{\mathrm{g}}$ then $\mathrm{i}\left(A\left(T_{\mathrm{n}}\right)\right)=\mathrm{n}$

\section{Proof:}

Let $a_{1}, a_{2}, \ldots, a_{n}$ be the vertices of $A\left(T_{n}\right)$ and $b_{i}$ be the new vertex joining alternatively $a_{i}$ and $a_{i+1}$ of a path by a triangle $C_{\mathrm{g}}$.

Now we select the vertices for the set $S$ (independent domination set) from a graph $A\left(T_{n}\right)$, in such a way that each vertex in $\mathrm{S}$ is adjacent to atleast one vertex in $\mathrm{V}-\mathrm{S}$ and that each vertex in $\mathrm{S}$ is independent.

Choosing such a way we get the independent domination number for $A\left(T_{n}\right)$ as (i.e.,)

$$
\mathrm{i}\left(A\left(T_{\mathrm{n}}\right)\right)=\mathrm{n}
$$

for example,

The highlighted vertices represent the independent domination set.

\section{Theorem 3:}

Let $D\left(T_{n}\right)$ be the double triangular snake obtained from two triangular snake with a common path then $\mathrm{i}\left(T_{n}\right)=\left\{\begin{array}{l}{\left[\frac{n+1}{2}\right] \text { when } n \text { is odd }} \\ {\left[\frac{n}{2}\right] \quad \text { when } n \text { is even }}\end{array}\right.$

Proof:

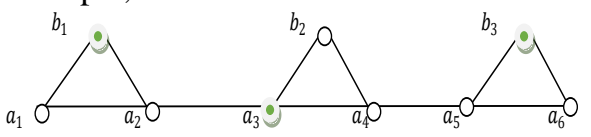

Let $a_{1}, a_{2}, \ldots, a_{n}$ be the vertices of $D\left(T_{n}\right)$ and $b_{i}$ be the new vertex joining $a_{i}$ and $a_{i+1}$ both above and below the common path of $a_{1}, a_{2, \ldots} a_{n}$.

Now we select the vertices for the set $S$ (independent domination set) from a graph $D\left(T_{n}\right)$, in such a way that each vertex in $\mathrm{S}$ is adjacent to atleast one vertex in $\mathrm{V}-\mathrm{S}$ and that each vertex in $\mathrm{S}$ is independent.

Choosing such a way we get the independent domination number for $D\left(T_{n}\right)$ as (i.e.,)

$$
\mathrm{i}\left(D\left(T_{n}\right)\right)=\left\{\begin{array}{l}
\left\lceil\frac{n+1}{2}\right] \text { when } n \text { is odd } \\
{\left[\frac{n}{2}\right] \quad \text { when } n \text { is even }}
\end{array}\right.
$$

for example, when $\mathrm{n}$ is odd,

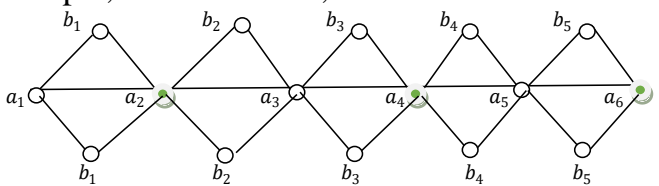

When $\mathrm{n}$ is even,

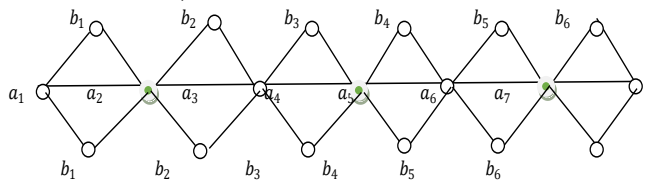

The highlighted vertices represent the independent domination set.

\section{Theorem 4:}

Let $D\left(A\left(T_{n}\right)\right)$ be the double alternative triangular snake obtained from alternate double triangular snake then $\mathrm{i}\left(D\left(A\left(T_{\mathrm{n}}\right)\right)\right)=\mathrm{n}$

\section{Proof:}

Let $a_{1}, a_{2}, \ldots a_{n}$ be the vertices of $D\left(A\left(T_{n}\right)\right)$ and $b_{i}$ be the new vertex joining alternatively $a_{i}$ and $a_{i+1}$ both above and below the common path of $a_{1}, a_{2, \ldots} a_{n}$.

Now we select the vertices for the set $S$ (independent domination set) from a graph $D\left(A\left(T_{n}\right)\right)$, in such a way that each vertex in $\mathrm{S}$ is adjacent to atleast one vertex in $\mathrm{V}-\mathrm{S}$ and that each vertex in $\mathrm{S}$ is independent.

Choosing such a way we get the independent domination number for $D\left(A\left(T_{n}\right)\right)$ as (i.e.,)

$$
\mathrm{i}\left(D\left(A\left(T_{\mathrm{n}}\right)\right)\right)=\mathrm{n}
$$

for example,

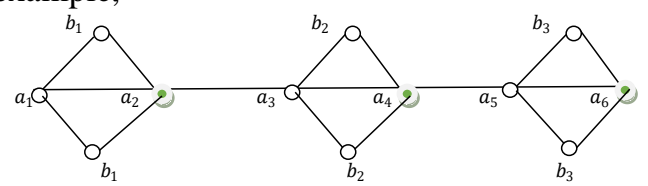

The highlighted vertices represent the independent domination set.

\section{Theorem 5:}

Let $Q_{\mathrm{n}}$ be the quadrilateral snake obtained from the path $P_{\mathrm{n}}$ by replacing each edge of the path by a cycle $C_{4}$ then $\mathrm{i}\left(Q_{n}\right)=$ $\mathrm{n}+1$

\section{Proof:}

Let $a_{1}, a_{2, \ldots} a_{n}$ be the vertices of $Q_{n}$ and $b_{i}$ and $c_{i}$ be the new vertex joining $a_{i}$ and $a_{i+1}$ of a path by a cycle $C_{4}$.

Now we select the vertices for the set $S$ (independent domination set) from a graph $Q_{n}$, in such a way that each vertex in $\mathrm{S}$ is adjacent to atleast one vertex in V-S and that each vertex in $\mathrm{S}$ is independent. 
Choosing such a way we get the independent domination number for $Q_{n}$ as (i.e.,)

for example,

$$
\mathrm{i}\left(Q_{\mathrm{n}}\right)=\mathrm{n}+1
$$

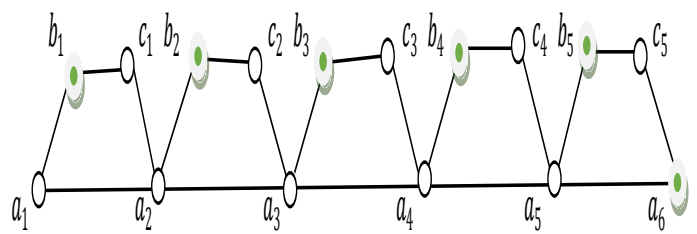

The highlighted vertices represent the independent domination set.

\section{Theorem 6:}

Let $A\left(Q_{n}\right)$ be the alternate quadrilateral snake obtained from the path $P_{\mathrm{n}}$ by replacing each alternate edge of the path by a cycle $C_{4}$ then $\mathrm{i}\left(A T_{n}\right)=\left\{\begin{array}{l}{\left[\frac{3 n+1}{2}\right\rceil \text { when } n \text { is odd }} \\ \left\lceil\frac{3 n}{2}\right\rceil \text { when } n \text { is even }\end{array}\right.$

Proof:

Let $a_{1}, a_{2}, \ldots, a_{n}$ be the vertices of $A\left(Q_{n}\right)$ and $b_{i}$ and $c_{i}$ be the new vertex joining alternatively $a_{i}$ and $a_{i+1}$ of a path by a cycle $C_{4}$.

Now we select the vertices for the set $S$ (independent domination set) from a graph $A\left(Q_{n}\right)$, in such a way that each vertex in $\mathrm{S}$ is adjacent to atleast one vertex in $\mathrm{V}-\mathrm{S}$ and that each vertex in $S$ is independent.

Choosing such a way we get the independent domination number for $A\left(Q_{n}\right)$ as (i.e.,)

$$
\mathrm{i}\left(A Q_{n}\right)=\left\{\begin{array}{l}
{\left[\frac{3 n+1}{2}\right\rceil \text { when } n \text { is odd }} \\
\left\lceil\frac{3 n}{2}\right\rceil \text { when } n \text { is even }
\end{array}\right.
$$

for example, when $\mathrm{n}$ is odd

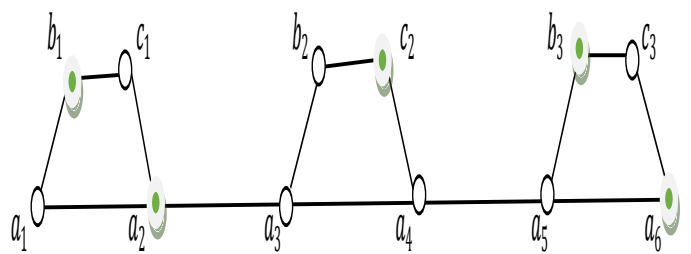

When $\mathrm{n}$ is even,

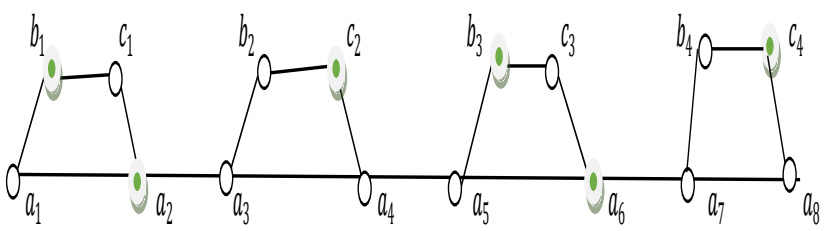

The highlighted vertices represent the independent domination set.

\section{Theorem 7:}

Let $D\left(Q_{\mathrm{n}}\right)$ be the double quadrilateral snake obtained from two quadrilateral snake with a common path then $\mathrm{i}\left(Q_{\mathrm{n}}\right)=2 \mathrm{n}$ Proof:

Let $a_{1}, a_{2}, \ldots, a_{n}$ be the vertices of $D\left(Q_{n}\right)$ and $b_{i}$ and $c_{i j}$ be the new vertex joining $a_{i}$ and $a_{i+1}$ both above and below the common path of $a_{1}, a_{2}, \ldots, a_{n}$.

Now we select the vertices for the set $S$ (independent domination set) from a graph $D\left(Q_{n}\right)$, in such a way that each vertex in $\mathrm{S}$ is adjacent to atleast one vertex in $\mathrm{V}-\mathrm{S}$ and that each vertex in $\mathrm{S}$ is independent.

Choosing such a way we get the independent domination number for $D\left(Q_{n}\right)$ as (i.e.,)

$$
\mathrm{i}\left(D\left(Q_{\mathrm{n}}\right)\right)=2 \mathrm{n}
$$

for example,

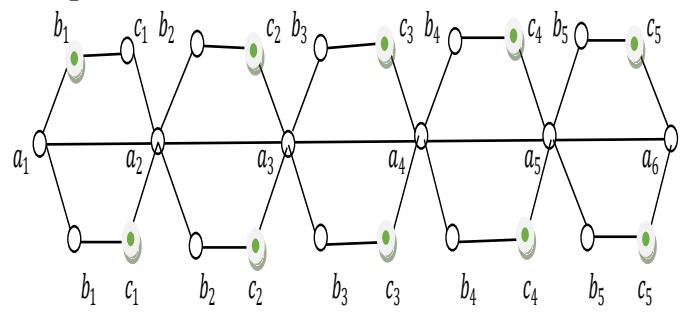

The highlighted vertices represent the independent domination set.

\section{Theorem 8:}

Let $D\left(A\left(Q_{n}\right)\right)$ be the double alternative quadrilateral snake obtained from alternate double quadrilateral snake then $\mathrm{i}\left(D\left(A\left(Q_{\mathrm{n}}\right)\right)\right)=2 \mathrm{n}$

\section{Proof:}

Let $a_{1}, a_{2}, \ldots a_{n}$ be the vertices of $D\left(A\left(Q_{n}\right)\right)$ and $b_{i}$ and $c_{i}$ be the new vertex joining alternatively $a_{i}$ and $a_{i+1}$ both above and below the common path of $a_{1}, a_{2 x} \ldots a_{n}$.

Now we select the vertices for the set $S$ (independent domination set) from a graph $D\left(A\left(Q_{n}\right)\right)$, in such a way that each vertex in $\mathrm{S}$ is adjacent to atleast one vertex in $\mathrm{V}-\mathrm{S}$ and that each vertex in $\mathrm{S}$ is independent.

Choosing such a way we get the independent domination number for $D\left(A\left(Q_{n}\right)\right)$ as (i.e., $)$

$$
\mathrm{i}\left(D\left(A\left(Q_{n}\right)\right)\right)=2 \mathrm{n}
$$

for example,

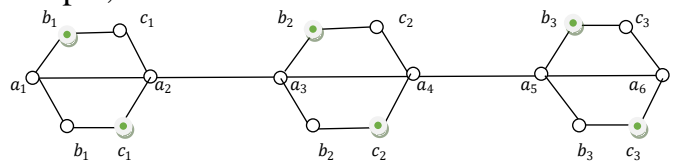

Note:

The independent domination number for $A\left(T_{n}\right)$ and $D\left(A\left(T_{n}\right)\right)$ is equal (i.e.,) n. Similarly, the independent domination number for $D\left(Q_{n}\right)$ and $A\left(Q_{n}\right)$ is equal (i.e.,) $2 n$.

\section{CONCLUSION}

We hereby found the independent domination number for triangular snake, alternate triangular snake, double triangular snake, alternate double triangular snake, quadrilateral snake, alternate quadrilateral snake, double quadrilateral snake and alternate double quadrilateral snake graphs.

\section{REFERENCES}

1. A. V. Kostochka, The independent domination number of a cubic 3 -connected graph can be much larger than its domination number, Graphs Combin., 9 (1993) pp. 235-237.

2. C. Berge . Theory of Graphs and its Applications, Methuen, London. (1962)

3. E. J. Cockayne, and S. T. Hedetniemi, Independent graphs, Congr Numer., X (1974) 471-491.

4. E. J. Cockayne and S. T. Hedetniemi. Towards a theory of domination in graphs, Networks 7 (1977) 247-261 
5. E. J. Cockayne, O. Favaron, H. Li, and G. MacGillivray, The product of the independent domination numbers of a graph and its complement, Discrete Mathematics, 90 (1991) 313-317.

6. J. Haviland. Upper bounds for independent domination in regular graphs, Discrete Math., 307 (2007) 2643-2646.

7. K.M.Baby Smitha and K.Thirusangu, Distance two labelling of quadrilateral snake families, 2(2016) 283-298.

8. O. Favaron. Two relations between the parameters of independence and irredundance, Discrete Math., 70 (1988) 17-20.

9. O. Ore, Theory of graphs, Amer. Math. Soc. Transl., 38 (1962) pp. 206-212.

10. P. Lam, W. Shiu and L. Sun On independent domination number of regular graphs, Discrete Mathematics Combin, 202 (1999) 135-144.

11. S. Ao, E. G. Cockayne MacGillivray and C. M. Mynhardt, Domination critical graphs with higher independent domination numbers, J. Graph Theory, 22 (1996) 9-14.

12. Sunoj B.S and Mathew Varkey T.K., Square difference prime labeling for some snake graphs, 3(2017) 1083-1089.

13. T.W. Haynes, S.M. Hedetniemi, S.T. Hedetniemi and M.A. Henning, Power domination in graphs applied to electrical power networks, SIAM Journal on Discrete Mathematics, 15(4) (2002) 519-529.

14. W. C. Shiu,X. Chen and W. H. Chan, Triangle-free graphs with large independent domination number, Discrete Optim., 7 (2010) pp. 86-92.

15. W.Goddard, M. Henning,J. Lyle and J. Southey. On the independent domination number of regular graphs, Ann. Comb., 16 (2012) 719-732.

16. Wilhelm Ahrens (1st ed.), Mathematische Unterhaltungen und Spiele, Teubner, Leipzig (1901)

\section{AUTHORS PROFILE}

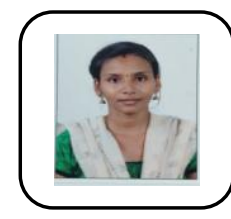

N.Senthurpriya Research scholar at Vels institute of science technology and advanced studies, pallavaram, Chennai-117, published a paper in The international journal of analytic and experimental modal analysis ISSN NO:0886-9367 under he topic Independent domination number in cycle necklace graph Vol XI Issue X October/2019.

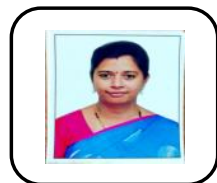

S.Meenakshi Associate Professor Department of Mathematics Vels institute of science technology and advanced studies, pallavaram, Chennai-117. 